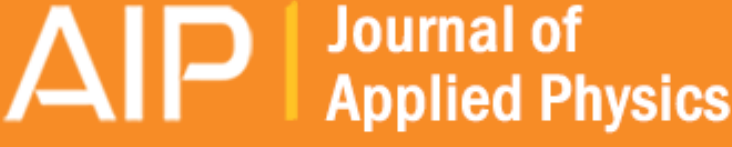

\section{Synthesis and characterization of Co2FeAl nanowires}

Keshab R. Sapkota, Parshu Gyawali, Andrew Forbes, lan L. Pegg, and John Philip

Citation: Journal of Applied Physics 111, 123906 (2012); doi: 10.1063/1.4729807

View online: http://dx.doi.org/10.1063/1.4729807

View Table of Contents: http://scitation.aip.org/content/aip/journal/jap/111/12?ver=pdfcov

Published by the AIP Publishing

\section{Articles you may be interested in}

Development and intrinsic properties of hexagonal ferromagnetic $(\mathrm{Zr}, \mathrm{Ti}) \mathrm{Fe} 2$

J. Appl. Phys. 115, 17A769 (2014); 10.1063/1.4868696

Magnetic and chemical order-disorder transformations in Co2Fe(Ga1-x Si x ) and Co2Fe(Al1-y Si y ) Heusler alloys

J. Appl. Phys. 111, 073909 (2012); 10.1063/1.3700220

Synthesis and magnetostriction of $\operatorname{Tb} x \operatorname{Pr} 1-x$ ( Fe 0.8 Co 0.2 ) 1.9 cubic Laves alloys

J. Appl. Phys. 105, 07A925 (2009); 10.1063/1.3073849

Structure and magnetocaloric effect in the pseudobinary system LaFe 11 Si 2 - LaFe $11 \mathrm{Al} 2$

J. Appl. Phys. 95, 6924 (2004); 10.1063/1.1667437

Structural and magnetic properties of Sm $2 \mathrm{Fe} \mathrm{17-x} \mathrm{T} \mathrm{x} \mathrm{M} \mathrm{(} \mathrm{T=Co,} \mathrm{Ti} \mathrm{;} \mathrm{M=Al,} \mathrm{Si} \mathrm{)} \mathrm{compounds}$ J. Appl. Phys. 85, 4672 (1999); 10.1063/1.370443

MIT LINCOLN

LABORATORY CAREERS

Discover the satisfaction of innovation and service

to the nation
- Space Control

- Air \& Missile Defense

- Communications Systems \& Cyber Security

- Intelligence, Surveillance and

Reconnaissance Systems

- Advanced
Electronics
- Tactical Systems
- Homeland
Protection
- Air Traffic Control

LINCOLN LABORATORY

MassachusetTs Institute of TeChNOLOgY

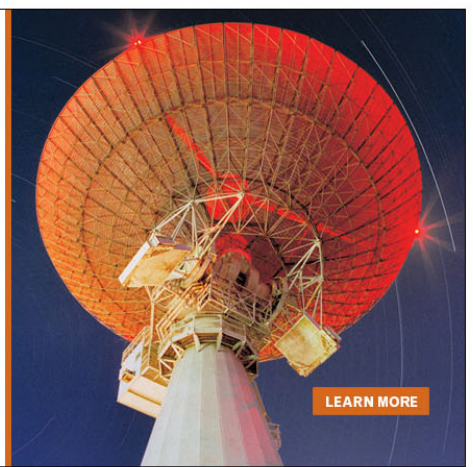




\title{
Synthesis and characterization of $\mathrm{Co}_{2} \mathrm{FeAl}$ nanowires
}

\author{
Keshab R. Sapkota, ${ }^{1,2}$ Parshu Gyawali, ${ }^{2}$ Andrew Forbes, ${ }^{2,3}$ Ian L. Pegg, ${ }^{1,2}$ \\ and John Philip ${ }^{1,2}$ \\ ${ }^{1}$ Department of Physics, The Catholic University of America, Washington, DC 20064, USA \\ ${ }^{2}$ The Vitreous State Laboratory, The Catholic University of America, Washington, DC 20064, USA \\ ${ }^{3}$ Department of Physics, Virginia Polytechnic Institute and State University, Blacksburg, Virginia 24061, USA
}

(Received 19 December 2011; accepted 21 May 2012; published online 19 June 2012)

\begin{abstract}
We report the growth and characterization of $\mathrm{Co}_{2} \mathrm{FeAl}$ nanowires. Nanowires are grown using electrospinning method and the diameters range from 50 to $500 \mathrm{~nm}$. These nanowires exhibit cubic crystal structure with a lattice constant of $a=5.639 \AA$. The nanowires exhibit ferromagnetic behavior with a very high Curie temperature. The temperature dependent magnetization behavior displays an anomaly in the temperature range $600-850 \mathrm{~K}$, which disappears at higher external magnetic fields. (C) 2012 American Institute of Physics. [http://dx.doi.org/10.1063/1.4729807]
\end{abstract}

\section{INTRODUCTION}

Ferromagnets with Curie temperature well-above room temperature are vital for fabricating novel spin-based electronic devices. ${ }^{1}$ Co-based Heusler alloys were extensively explored in the literature and some of them are shown to be half-metallic. ${ }^{1-4}$ High Curie temperatures were observed in Co-based Heusler compounds. $\mathrm{Co}_{2} \mathrm{FeAl}$ (CFA) is a full Heusler alloy; the half-metallic behavior of CFA is not yet clearly understood. There are calculations showing both the presence and the absence of half-metallicity in CFA. ${ }^{1-4} \mathrm{CFA}$ thin film is reported to occur in various structures ranging from the completely ordered $\mathrm{L} 22_{1}$, moderately ordered B2, and completely disordered A2 structure types. ${ }^{5}$ Spin polarization in two-dimensional CFA structures is reported to be around $56 \%$ (Refs. 6 and 7) measured by the point contact Andreev reflection method. Also, tunneling magnetoresistance for CFA junctions with a $\mathrm{MgO}$ tunnel barrier is reported around $330 \%$ at room temperature irrespective of the $\mathrm{L} 2{ }_{1}$ or B2 structure. ${ }^{8}$ Bulk and two-dimensional structures of CFA have been studied extensively and reported in the literature. One-dimensional structures of CFA have not yet been explored. In this work, we report the growth, characterization, and magnetic properties of CFA nanowires.

\section{PREPARATION OF NANOWIRES}

CFA nanowires are grown by the electrospinning method. Nitrates of $\mathrm{Co}, \mathrm{Fe}$, and $\mathrm{Al}$ are mixed in the right ratio in deionized water and added to a mixture of polyvinyl alcohol (PVA) and polyvinylpyrrolidone (PVP) solution for electrospinning. The viscosity of the solution is adjusted using the ratio of the polymer, which determines the diameter of nanowires. 9,10 Continuous fibers are formed on the Si/quartz substrate by an electrospinning process ${ }^{11}$ and then annealed at $1023 \mathrm{~K}$ for $3 \mathrm{~h}$ in ultrahigh purity Ar with $3 \%$ hydrogen gas mixture. In the annealing process, PVA and PVP were removed, nitrates were decomposed, and continuous CFA nanowires were formed.

\section{CHARACTERIZATION OF NANOWIRES}

The morphology of CFA nanowires is shown in Figure 1. The scanning electron microscopy image (Figure 1(a)) shows dense nanowires with diameter of wires range from 50 to $500 \mathrm{~nm}$. Figure 1(b) displays the morphology of single nanowire obtained using transmission electron microscopy (TEM). Nanowires are continuous and granular in nature. The crystal structure of CFA nanowires is investigated by X-ray diffraction (XRD) with $\mathrm{Cu} K$ - $\alpha$ radiation. The observed XRD pattern shows three peaks with $h k l$ values (200), (220), and (400) as shown in Figure 2. XRD patterns of full Heusler alloys can be divided into odd superlattice, even superlattice, and fundamental diffractions. ${ }^{12,13}$ For odd superlattice diffraction, peaks are obtained at odd $h k l$ values. Only the L $2_{1}$ crystal structure displays odd superlattice diffraction. For even superlattice diffraction, peaks are obtained at those $h \mathrm{kl}$ values satisfying $h+k+l=4 n+2$, where $\mathrm{n}$ is a positive integer. Both B2 and L2 1 structures show even superlattice diffraction. For fundamental diffraction, $h k l$ values satisfy the relation $h+k+l=4 n$. This type of diffraction is shown by $\mathrm{A} 2, \mathrm{~B} 2$, and $\mathrm{L} 2{ }_{1}$ types of crystal structures. So, the observed peaks of CFA nanowires at (200), (220), and (400) correspond to a B2 structure. The comparison of intensities at (200) and (400) with intensity at (220) suggests that wires may consist of B2 as well as A2 types of crystal structures. The lattice constant of CFA nanowires obtained from XRD analysis is $a=5.639 \AA$, which is within $1 \%$ of the lattice parameter for the bulk system with $\mathrm{L} 2{ }_{1}$ structure. It is reported that the B2 structure can have $10 \%$ variation in lattice parameter with respect to the $\mathrm{L} 2{ }_{1}$ structure. ${ }^{14}$ In order to get more insight into the structure of CFA nanowires, more TEM analyses are carried out. High resolution TEM micrograph in Figure 3(a) (inset) clearly shows the granular nature of the nanowires. The grain size varies from 10 to $40 \mathrm{~nm}$. The lattice fringes shown in Figure 3(a) can be indexed based on the cubic crystal structure of CFA. The observed lattice fringes correspond to (200) planes. The selected area electron diffraction pattern in Figure 3(b) displays the polycrystalline nature of CFA nanowires. The rings in the pattern can be indexed to (200), (220), (400), and (422) planes of CFA structure. ${ }^{15}$

The magnetic measurements of the as-grown wire ensemble were investigated using a vibrating sample magnetometer from 2 to $1000 \mathrm{~K}$. Figure 4 shows the magnetic 


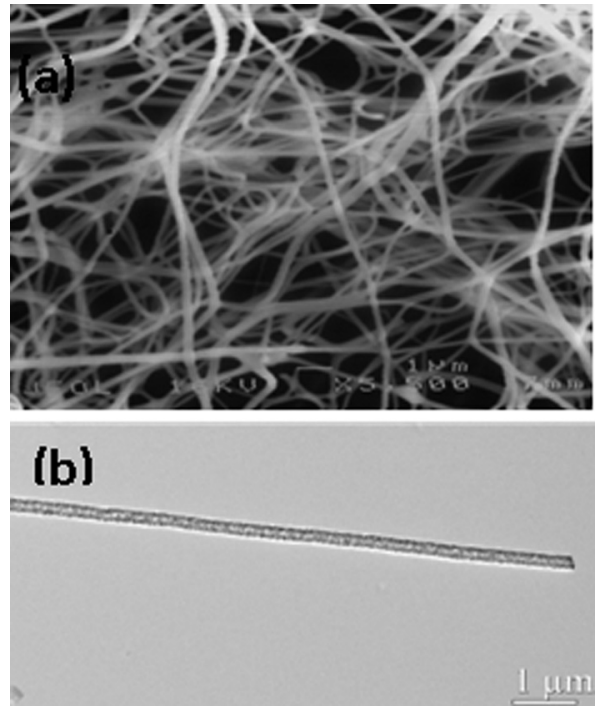

FIG. 1. (a) Scanning electron microscopy image of CFA nanowires. (b) TEM micrograph of a single CFA nanowire with diameter less than $100 \mathrm{~nm}$.

properties of CFA nanowires that were measured with the magnetic field applied parallel to the substrate plane. The high temperature magnetization behavior shows that the magnetic transition temperature of the CFA nanowires is too high to observe with our present experimental setup. The shape of the temperature dependence above $600 \mathrm{~K}$ is rather unusual with a cusp around $650 \mathrm{~K}$. The hysteresis loops in the inset of Figure 4 show that the magnetic moment increases from 650 to $800 \mathrm{~K}$ in contrast to the behavior observed in regular ferromagnets. This behavior is also observed in bulk CFA; it is hypothesized that the presence of a partial ferrimagnetic order or a partial antiparallel coupling exists at different sites at elevated temperatures. ${ }^{16}$ Another possibility is that the nanowires undergo a structural transition around this temperature. In order to understand whether there is any structural change in CFA in the temperature range $600-1000 \mathrm{~K}$, we have prepared CFA nanowires and analyzed them using the differential scanning calorimetry. There is no structural transition observed as displayed in Figure 5. The heating and the cooling curves do not show any anomaly in the temperature range $300-1100 \mathrm{~K}$.

In order to understand the high temperature magnetic behavior, we have measured magnetization as a function of

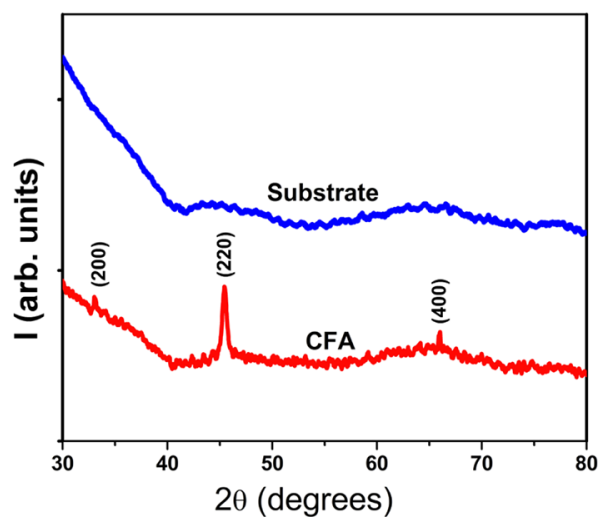

FIG. 2. The XRD patterns of CFA nanowires and the quartz substrate.

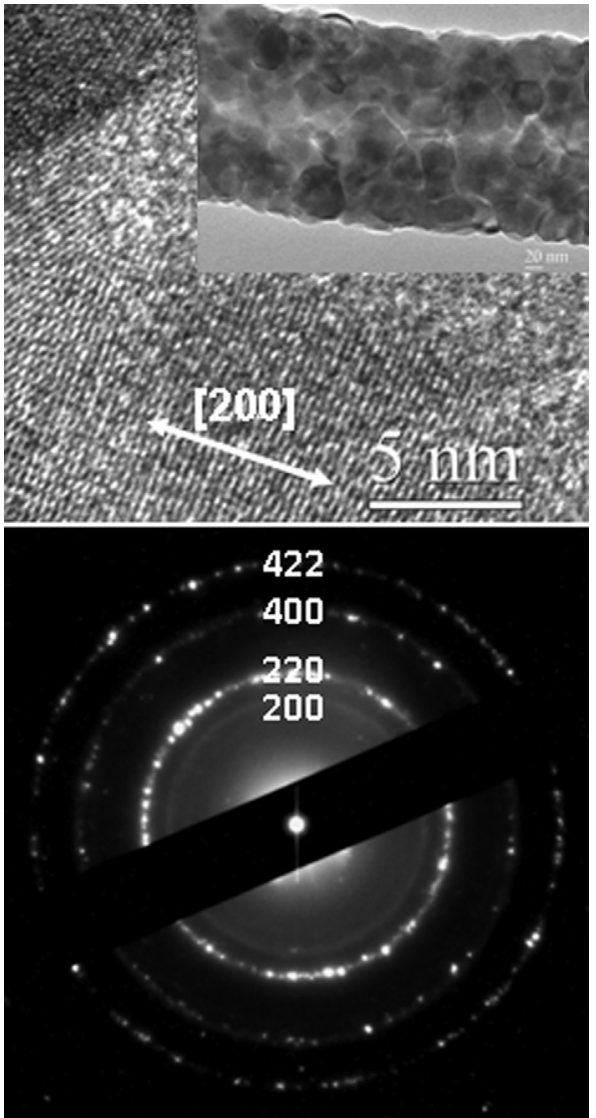

FIG. 3. (a) High resolution TEM image displaying the lattice fringes. Displayed lattice fringes are in the [200] direction. Inset shows the granular nature of a nanowire. (b) Selected area electron diffraction of a CFA nanowire showing the polycrystalline nature of the wires.

temperature with field-cooling and with external magnetic fields applied during measurements. It is observed that if we field-cool the nanowires from 1000 to $300 \mathrm{~K}$ in the presence of an external field greater than $2 \mathrm{kOe}$ and measure the $\mathrm{M}$ vs. $\mathrm{T}$ at the same field, then the cusp at higher temperature

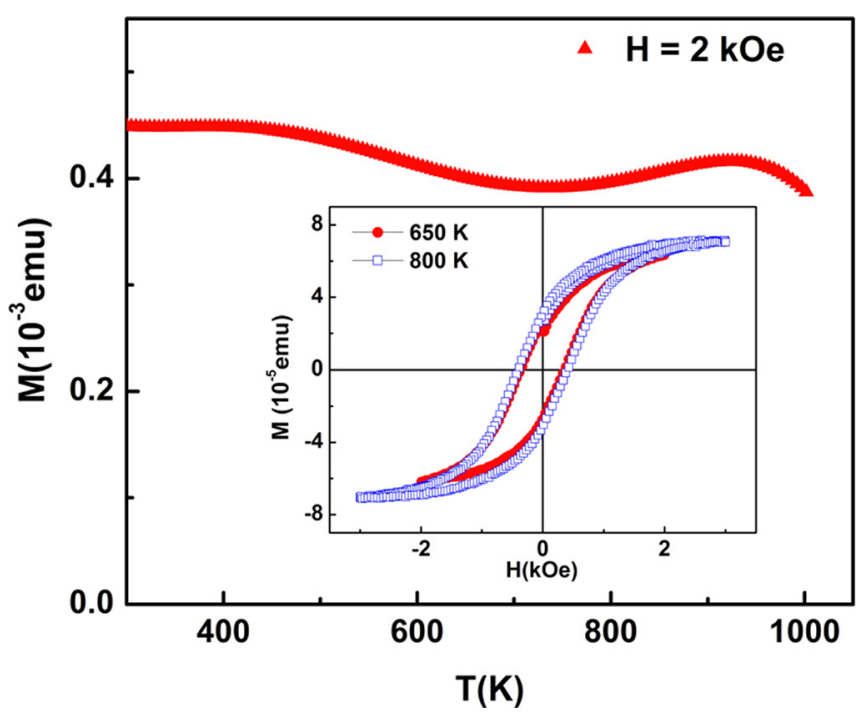

FIG. 4. The magnetization versus temperature plot shows cusp around $650 \mathrm{~K}$ at $2 \mathrm{kOe}$ external magnetic field. Inset shows the hysteresis loops of CFA nanowires at $650 \mathrm{~K}$ and $800 \mathrm{~K}$. 


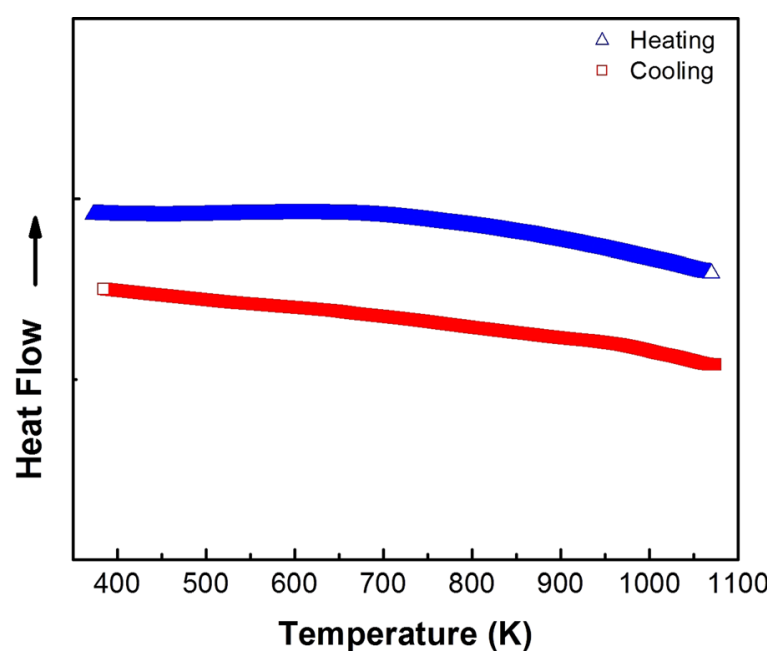

FIG. 5. Differential scanning calorimetry analyses show that there is no phase transition in the temperature range $300-1100 \mathrm{~K}$ in CFA nanowires.

disappears. Figure 6 shows that the cusp in M vs. T curves shifts to higher temperatures with the increase in external magnetic field and finally disappears above $2 \mathrm{kOe}$. This clearly shows that the anomaly in M vs. $\mathrm{T}$ is observed only when measurements are carried out with zero or small external magnetic field strengths. The observed cusp in the $\mathrm{M}$ vs $\mathrm{T}$ curves can be explained on the basis of the granular nature of the nanowires. Each grain has local axis of magnetization, say easy axis. ${ }^{17}$ Since CFA nanowires are polycrystalline, the magnetization in the grains is randomly oriented (anisotropy). For large anisotropy and for small external magnetic field, the magnetization vector will stay about easy axis. So, at low applied magnetic fields, initial contribution on $\mathrm{M}$ vs $\mathrm{T}$ measurement is from all grains where the magnetization is aligned in all possible directions. As temperature is increased, magnetization shows regular ferromagnetic behavior until to a temperature at which a few misaligned (with respect to the applied field) grains are weakened enough by thermal energy and the magnetization vectors are rotated along the external field. The misaligned grains with least energy barrier to reversal, which depends on the shape and crystallographic anisotropy, volume, easy axis orientation of the grain with respect to

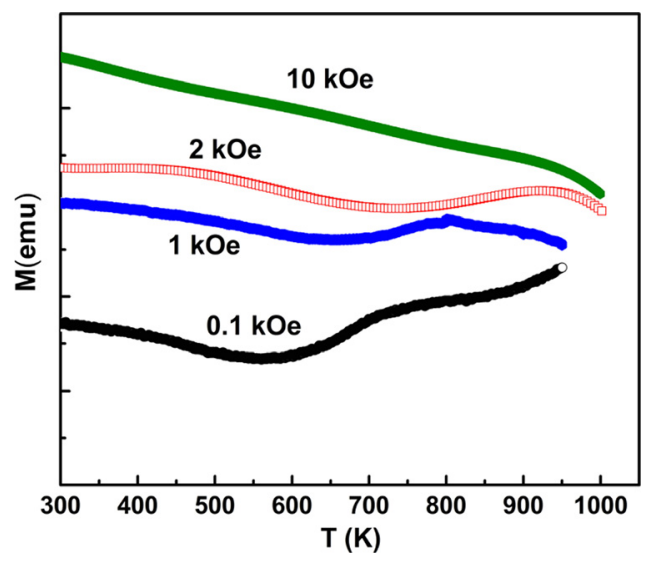

FIG. 6. Magnetization versus temperature plots for different applied magnetic fields. Plots show that the cusp disappears at high external magnetic field.

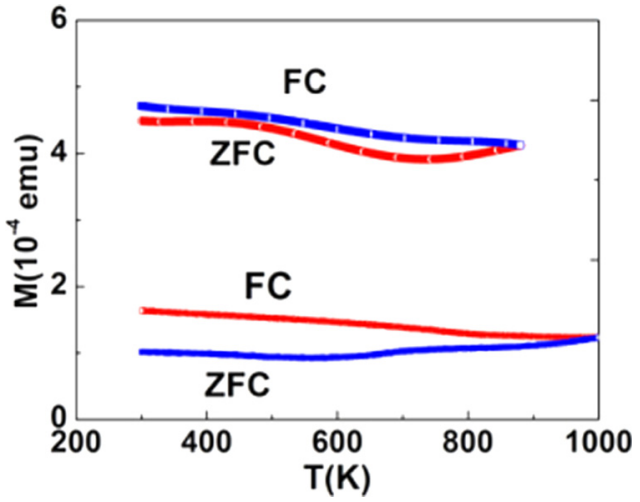

FIG. 7. The zero-field-cooled and field-cooled magnetization measurements carried out at 0.1 (lower) and at $2 \mathrm{kOe}$ (upper).

external field and interaction among grains, will be switched initially along the external field direction. ${ }^{18,19}$ As temperature is increased, more grains will be switched in the direction of the field depending upon the magnitude of the field. Hence, the magnetization starts to increase because more domains are aligned in the direction of the external field, though each domain contribution to magnetization has decreased due to thermal demagnetization effect. The magnetization attains a peak value at a certain higher temperature and afterward, the M vs $\mathrm{T}$ exhibits regular ferromagnetic behavior. If we apply high external magnetic field ( $>2 \mathrm{kOe}$ ), it is sufficiently strong to align the magnetization vectors in all the grains irrespective of the easy axis of the grains; therefore, the M vs T measurement shows no cusp. This is also supported by the $\mathrm{M}$ vs $\mathrm{H}$ plots in Figure 4, which shows that the magnetization is saturated above $2 \mathrm{kOe}$. The field-cooled (FC) and zero-field-cooled (ZFC) measurements shown in Figure 7 display that at low fields, there is a large difference between the FC and ZFC curves, but near $2 \mathrm{kOe}$, the difference reduces and above 2 $\mathrm{kOe}$, these two curves merge together. These ZFC and FC behaviors can also be explained on the basis of granular nature of the nanowires.

\section{CONCLUSIONS}

We have presented the growth, structural characterization, and magnetic properties of the Heusler alloy $\mathrm{Co}_{2} \mathrm{FeAl}$ nanowires. Nanowires with a diameter in the range of $50-500 \mathrm{~nm}$ can be consistently grown. The nanowires are continuous with granular surfaces. The magnetic behavior of CFA nanowires exhibits ferromagnetic behavior with a high Curie temperature.

\section{ACKNOWLEDGMENTS}

This work was supported by National Science Foundation under ECCS-0845501 and NSF-MRI, DMR-0922997. We thank Cathy Paul for carefully reading the manuscript.

${ }^{1}$ K. Inomata, N. Ikeda, N. Tesuka, R. Goto, S. Sugimoto, M. Wojcik, and E. Jedryka, Sci. Technol. Adv. Mater. 9, 014101 (2008).

${ }^{2}$ Y. Miura, K. Nagao, and M. Shirai, Phys. Rev. B 69, 144413 (2004).

${ }^{3}$ T. M. Nakatani, A. Rajanikanth, Z.Gercsi, Y. K. Takahashi, K. Inomata, and K. Hono, J. Appl. Phys. 102, 033916 (2007). 
${ }^{4} \mathrm{X}$. Xu, Y. Wang, D. Zhang, and Y. Jiang, in 1st International Symposium on Spintronic Devices and Commercialization (ISSDC2010), Beijing, China, 21-24 October 2010 [J. Phys.: Conf. Ser. 263, 012016 (2011)].

${ }^{5}$ S. Wurmehl, J. T. Kohlhepp, H. J. M. Swagten, and B. Koopmans, J. Phys. D: Appl. Phys. 41, 115007 (2008).

${ }^{6}$ S. V. Karthik, A. Rajanikanth, Y. K. Takahashi, T. Okhubo, and K. Hono, Appl. Phys. Lett. 89, 052505 (2006).

${ }^{7}$ O. Schebaum, D. Ebke, A. Niemeyer, G. Reiss, J. S. Moodera, and A. Thomas, J. Appl. Phys. 107, 09C717 (2010).

${ }^{8}$ W. Wang, E. Liu, M. Kodzuka, H. Sukegawa, M. Wojcik, E. Jedryka, G. H. Wu, K. Inomata, S. Mitani, and K. Hono, Phys. Rev. B 81, 140402(R) (2010).

${ }^{9}$ S. Ramakrishna, K. Fujihara, W. E. Teo, T. C. Lim, and Z. Ma, An Introduction to Electrospinning and Nanofibers (World Scientific, Singapore, 2005).

${ }^{10}$ S. A. Theron, E. Zussman, and A. L. Yarin, Polymer 45, 2017-2030 (2004).
${ }^{11}$ W. E. Teo and S. Ramakrishna, Nanotechnology 17, R89-R106 (2006).

${ }^{12}$ P. J. Webster, J. Phys. Chem. Solids 32, 1221 (1971).

${ }^{13}$ Y. Takamura, R. Nakane, and S. Sugahara, J. Appl. Phys. 107, 09B111 (2010).

${ }^{14}$ J. Schmalhorst, M. Sacher, A. Thomas, H. Brückl, G. Reiss, and K. Starke, J. Appl. Phys. 97, 123711 (2005).

${ }^{15}$ S. Okamura, A. Miyazaki, S. Sugimoto, N. Tezuka, and K. Inomata, Appl. Phys. Lett. 86, 232503 (2005).

${ }^{16}$ V. Jung, G. H. Fecher, B. Balke, V. Ksenofontov, and C. Felser, J. Phys. D: Appl. Phys. 42, 084007 (2009).

${ }^{17}$ H. J. Elmers, S. Wurmehl, G. H. Fecher, G. Jakob, C. Felser, and G. Schonhense, Appl. Phys. A: Mater. Sci. Process. 79, 557-563 (2004).

${ }^{18}$ A. Winter, H. Pascher, H. Krenn, X. Liu, and J. K. Furdyna, J. Appl. Phys. 108, 043921 (2010).

${ }^{19}$ S. J. Lister, T. Thomson, J. Kohlbrecher, K. Takano, V. Venkataramana, S. J. Ray, M. P. Wismayer, M. A. de Vries, H. Do, Y. Ikeda, and S. L. Lee, Appl. Phys. Lett. 97, 112503 (2010). 